\title{
A misplaced and entrapped pulmonary artery catheter
}

\author{
Soo-Kyung Park ${ }^{1}$, Nam-Su Gil ${ }^{2}$, Anna Kim ${ }^{1}$, and Ho-Geol Ryu ${ }^{1}$ \\ Department of Anesthesiology and Pain Medicine, ' Seoul National University Hospital, ${ }^{2}$ Boramae Medical Center, Seoul, Korea
}

In 1970, Swan et al. [1] reported that a pulmonary capillary wedge pressure could be obtained by cardiac catheterization without the aid of fluoroscopy. Since then, the Swan-Ganz pulmonary artery catheter has been widely used to monitor hemodynamic changes and cardiac output in critically ill patients. A pulmonary artery catheter is placed through a large-bore introducer sheath and the right internal vein is preferred because of its straight course to the right atrium and right ventricle. Recently, we encountered a patient, in whom entrapment of the pulmonary artery catheter occurred as it entered the right ventricle, leading to catheter function failure. The patient required surgical removal of the catheter.

A 66-year-old male was admitted for a transient ischemic attack. The patient had a history of hypertension and type II diabetes mellitus. The brain MRI/MRA revealed a total occlusion of the right proximal internal carotid artery, severe stenosis in the right proximal external carotid artery, and an atheromatous plaque with moderate narrowing of the left proximal internal carotid artery. To evaluate the cerebral vessels, a transfemoral cerebral angiography (TFCA) was performed. The patient had a mild stenotic artery with an ulcer in the left internal carotid artery. He was scheduled for a left carotid endarterectomy. To evaluate his heart function before surgery, a myocardial single-photon emission computed tomography was performed. A perfusion decrease in the basal anterior wall was found. A coronary angiography confirmed diffuse three vessel disease. After consultation between neurosurgery and cardiothoracic surgery, the patient was scheduled to undergo a simultaneous carotid endarterectomy and coronary artery bypass graft surgery.

Before the induction of general anesthesia, the continuous arterial pressure was monitored by placing a 20 -guaged catheter in the right radial artery. An induction was done using $5 \mathrm{mg}$ of midazolam, $6 \mathrm{mg}$ of etomidate, and $10 \mathrm{mg}$ of vecuronium. After endotracheal intubation, an advanced venous access (AVA) catheter was placed in the right internal jugular vein under ultrasound guidance. Afterwards, the Swan-Ganz catheter (SGC) was inserted with the catheter tip facing the 9 o'clock direction. The catheter advanced smoothly up to $70 \mathrm{~cm}$, but failed to reach the pulmonary artery and could not be advanced further. In the subsequent attempt, the catheter was inserted to $70 \mathrm{~cm}$, but it was also unsuccessful.

Resistance was felt when trying to withdraw the catheter for a third insertion attempt. The catheter withdrawal was immediately stopped, and a chest radiograph was performed and checked in the operating room. The chest radiograph showed an acute angulation of the SGC at the tip of the AVA catheter. Radiology consultation confirmed that the tip of the SGC was in the left azygous vein. There was no arrhythmia and the arterial pressure was stable throughout the insertionwithdrawal-reinsertion procedure.

Following a successful carotid endarterectomy, the cardiac surgeon began coronary artery bypass graft surgery. After initiating a cardiopulmonary bypass, the SGC was retrieved. There was a small notch at the $40 \mathrm{~cm}$ mark of the catheter, but no evidence of knotting (Fig. 1).

Several complications are associated with the insertion and presence of a SGC. A previous report showed that among 230 SGCs inserted, 9 (3.9\%) were associated with major complications including a knotted catheter that had to be surgically removed [2]. Other reported complications include severe dysrhythmias (ventricular tachycardia or fibrillation)

Corresponding author: Ho-Geol Ryu, M.D., Department of Anesthesiology and Pain Medicine, Seoul National University Hospital, 101, Daehag-ro, Jongro-gu, Seoul 110-744, Korea. Tel: 82-2-2072-2065, Fax: 82-2-747-5639, E-mail: hogeol@gmail.com

(c) This is an open-access article distributed under the terms of the Creative Commons Attribution Non-Commercial License (http:// creativecommons.org/licenses/by-nc/3.0/), which permits unrestricted non-commercial use, distribution, and reproduction in any medium, provided the original work is properly cited. 


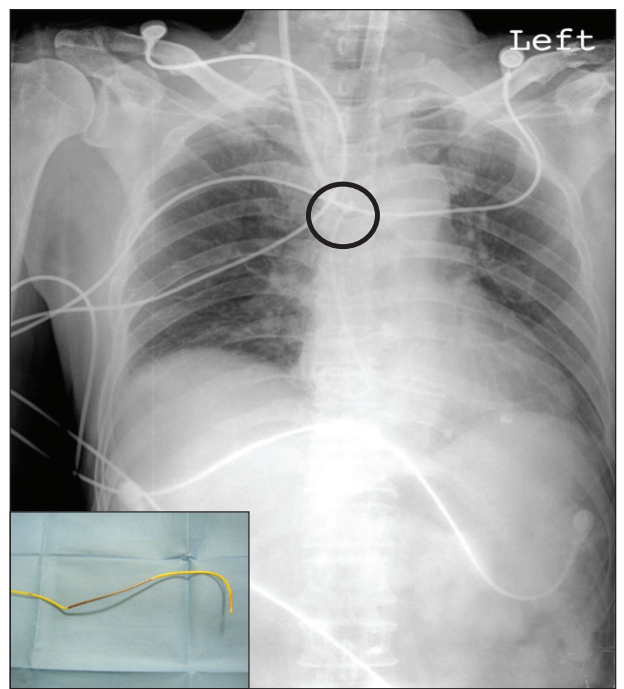

Fig. 1. Chest X-ray after pulmonary artery catheter placement and the retrieved pulmonary artery catheter. The chest X-ray shows an acute angle bending of the pulmonary catheter. The picture in the left lower corner shows the retrieved pulmonary artery catheter with a dent at about $25 \mathrm{~cm}$ from the tip.

(0.3-62.7\%), a small increase in tricuspid regurgitation (17\%), a right bundle-branch block $(0.1-4.3 \%)$, and complete heart block in patients with prior left bundle-branch block (0-8.5\%). Complications related to the catheter itself include pulmonary artery rupture $(0.03-1.5 \%)$, catheter related infections (1.4$34.8 \%)$, catheter-related sepsis $(0.7-11.4 \%)$, thrombophlebitis (6.5\%), venous thrombosis $(0.5-66.7 \%)$, pulmonary infarction $(0.1-5 \%)$, mural thrombus $(28-61 \%)$, valvular or endocardial vegetations or endocarditis $(2.2-100 \%)$, and even death $(0.02-$ $1.5 \%)[3]$.

In our case, the anteroposterior chest radiograph suggested that the SGC was knotted. The catheter, however, turned out to be just trapped. Benson and Patla [4] suggested that the distinction could be made by sliding the introducer sheath over the catheter and continuing to withdraw the sheath and catheter from the patient. If the catheter still is trapped, it is most likely caught somewhere in the heart or the great vessels.
We suspect that the reason for the catheter's entrapment was due to the twisting force applied to the catheter for insertion into the pulmonary artery. The thermodilution section of the wire is stiffer than other parts of the SGC, and during withdrawal of the SGC, the area just above the thermodilutional wire was bent (Fig. 1).

Surgical procedures are usually performed for the removal of large sized knots and loops, intracardiac fixing of the SGC, and suture entrapments of the SGC. Depending on the entrapped site, subclavian venotomy, jugular venotomy, femoral venotomy, or cardiotomy can be done. Even though this case was different from the above mentioned cirucumstances, a sternotomy and cardiopulmonary bypass was electively done for the coronary bypass graft operation and the cardiac surgeon performed an atriotomy and removed the catheter.

Nonsurgical removals have resolved many knots and loop problems for more than 30 years by radiologists. Interventional radiologic techniques have been invented to untie knotted catheters and to retrieve embolized catheter fragments. With the internal jugular vein approach, the deflection wire can be used. The wire is locked in the first turn of the SGC and the catheter is then withdrawn and the folded part of the catheter slipping over the wire is released [5].

\section{References}

1. Swan HJ, Ganz W, Forrester J, Marcus H, Diamond G, Chonette D. Catheterization of the heart in man with use of a flow-directed balloon-tipped catheter. N Engl J Med 1970; 283: 447-51.

2. Slung HB, Scher KS. Complications of the Swan-Ganz catheter. World J Surg 1984; 8: 76-81.

3. Practice guidelines for pulmonary artery catheterization: an updated report by the American Society of Anesthesiologists Task Force on Pulmonary Artery Catheterization. Anesthesiology 2003; 99: 9881014.

4. Benson J, Patla V. Real or apparent entrapment of a Swan-Ganz pulmonary artery catheter after cardiac surgery? Intensive Care Med 1994; 20: 309-10.

5. Hawkins IF, Tonkin A. Deflector method for nonsurgical removal of knotted catheters. Radiology 1973; 106: 705. 